\title{
Acute Urography: a Method for General Use
}

\author{
R. D. W. McLEAN,* F.R.C.S.; H. M. BENNETT, † F.R.C.S.; D. G. C. WHYTE, $\ddagger$ M.D., F.R.C.P.ED., F.R.C.P.GLASG.
}

\author{
[With Special Plate]
}

Brit. med. F., 1969, 1, 142-144

Cummary : A new method of acute urography suitable Sfor use in any general hospital is described. It takes only 30 minutes and can be performed by the duty radiographer.

The method has proved invaluable in the diagnosis and differentiation of renal colic. With radiation safeguards, it may be of value in pregnancy. It has been found useful in renal trauma and in the differential diagnosis of haematuria.

\section{Introduction}

Acute urography is a method of intravenous pyelography performed during renal colic or other symptom referable to the renal tract, without prior dehydration or bowel preparation. This technique was introduced to clinical practice over 30 years ago by Hellmer (1935) and Wulff (1935). Since then its value has been confirmed in the diagnosis and management of ureteric calculus (Wesson and Fulmer, 1932 ; Woodruff and Lewis, 1941 ; Heilbrun and Chittenden, 1946; Elkin, 1963 ; Goy, 1963; Smith, 1966) in the differential diagnosis of the acute abdomen (Ross et al., 1962), and in renal trauma (Nunn, 1962). Nevertheless, the use of acute urography in Britain seems to be very limited, and it is not yet a routine investigation. One reason for this may be that most of the methods described have taken from 4 to 24 hours to complete and require the supervision of a radiologist.

We have developed a method of acute urography which takes 30 minutes and which can be performed easily by the duty radiographer. This method may be used routinely by any general hospital admitting acute emergency cases. We have used it for a year, and our findings are reported below.

\section{Patients Studied and Methods}

A total of 67 patients underwent acute urography. In 53 the indications were " renal colic," in 7 haematuria following trauma, and in 7 painless haematuria. In all cases the investigation was done as soon as the diagnosis was suspected and while the patient had symptoms.

For the examination $40 \mathrm{ml}$. of $60 \%$ iodine solution was injected intravenously ( $80 \mathrm{ml}$. if the patient was obese). All radiographs measured 17 by 14 in. ( 43 by $35.5 \mathrm{~cm}$.) and included the whole renal tract; besides the preliminary film, additional films were taken at 5 and 15 minutes, and subsequently if requested by the clinician. (Factors used were: normal, $60 \mathrm{kV}, 200 \mathrm{~mA}, 0.3 \mathrm{sec}$. Bucky, fast film and screens ; and portable, $65 \mathrm{kV}, 100 \mathrm{~mA}, 1$ sec. Bucky, fast film and screens.)

\section{Results}

Urography confirmed the presence of renal disease in 44 of the 53 patients with renal colic. Obstructed kidney was shown in 42 , other renal disease in two, while normal urograms were obtained in nine. The radiographic appearances in patients with an obstructed kidney fell into one of the well-defined groups: obstructed kidney, ureter outlined, and calculus demonstrated in ureter, 23 cases (Special Plate, Fig. 1);

* Senior Registrar, Altnagelvin Hospital, Londonderry.

+ Consultant Surgeon, Altnagelvin Hospital, Londonderry.

$\neq$ Consultant Radiologist, Altnagelvin Hospital, Londonderry. obstructed kidney, ureter not outlined, opaque shadow in line of ureter, 15 cases (Special Plate, Fig. 2); and obstructed kidney, ureter not outlined, no opaque shadow seen in line of ureter (nephrogram effect), four cases (Special Plate, Fig. 3). The ureteric obstruction was found to be due to a ureteric calculus in all but two cases, one being caused by malignant lymph nodes and the other by carcinoma of the bladder.

The final diagnosis in the nine patients who were suspected clinically of having renal colic but had normal acute urograms is shown in the Table.

Final Diagnosis in Nine Patzents with Normal Urograms $\begin{array}{llllllll}\text { Retrocaecal appendicitis } & \ldots & 3 & \text { Torsion of ovarian cyst } & \ldots & 1 \\ \text { Diverticular disease of colon } & \ldots & 2 & \begin{array}{l}\text { Perforation of duodenal ulcer } \\ \text { Biliary colic }\end{array} \ldots & \ldots & 1 & 1\end{array}$

Seven patients presented with painless haematuria, and without other urinary symptoms. The diagnosis was made on acute urography in three of these (one each with neoplasm of kidney, neoplasm of bladder, and diverticulum of bladder). Four had normal urograms, the diagnosis being made after further investigation.

\section{Discussion}

Despite many of the radiographs being taken by relatively inexperienced radiographers, often in the early hours of the morning, the quality of the radiographs was good and all were adequate for interpretation. Concentration of the medium in the contralateral kidney was adequate despite normal hydration. The presence of gas in the intestines was not a problem. This confirms the impression of Smith (1966) that urograms of patients taken "off the street" are superior to those of inpatients subjected to formal preparation. Investigation did not prove upsetting to patients provided analgesia was given, and took little more in time and effort than a plain radiograph of the abdomen.

Obstructed Kidney.-The appearances on urography when there is obstruction to urinary flow down the ureter have been described by Wulff (1935), Hellmer (1935), and Boeminghaus (1932). Obstruction leads to a back-pressure effect and produces the following sequence of events: (1) delay in appearance of the contrast medium on the affected side; (2) the medium being held in the tubules-nephrogram effect ; (3) delay in outlining the calices, pelvis, and ureter ; (4) dilatation of the pelvis and ureter; and (5) delay in evacuation of pelvis and ureter. The rate at which this sequence develops will depend on the degree of obstruction and the length of time this has been present (Elkin, 1963). Hence a radiograph taken at any time after the injection of contrast medium may show any one of the above appearances and indicate ureteric obstruction.

Our findings on the causes of renal obstruction support the views of others practising acute urography-namely, that colic and an obstructed kidney are in the vast majority of cases due to stone, other causes being rare. It should be noted that some patients were not aware that they had in fact passed a calculus ; others expected a "kidney stone" to be much larger than "the piece of grit" which they passed, and which they often discounted.

\section{Value of Acute Urography}

The value of acute urography in renal colic lies in the high incidence of positive diagnoses. Wulff (1935) found that when 
R. D. W. MCLEAN ET AL.: ACUTE UROGRAPHY

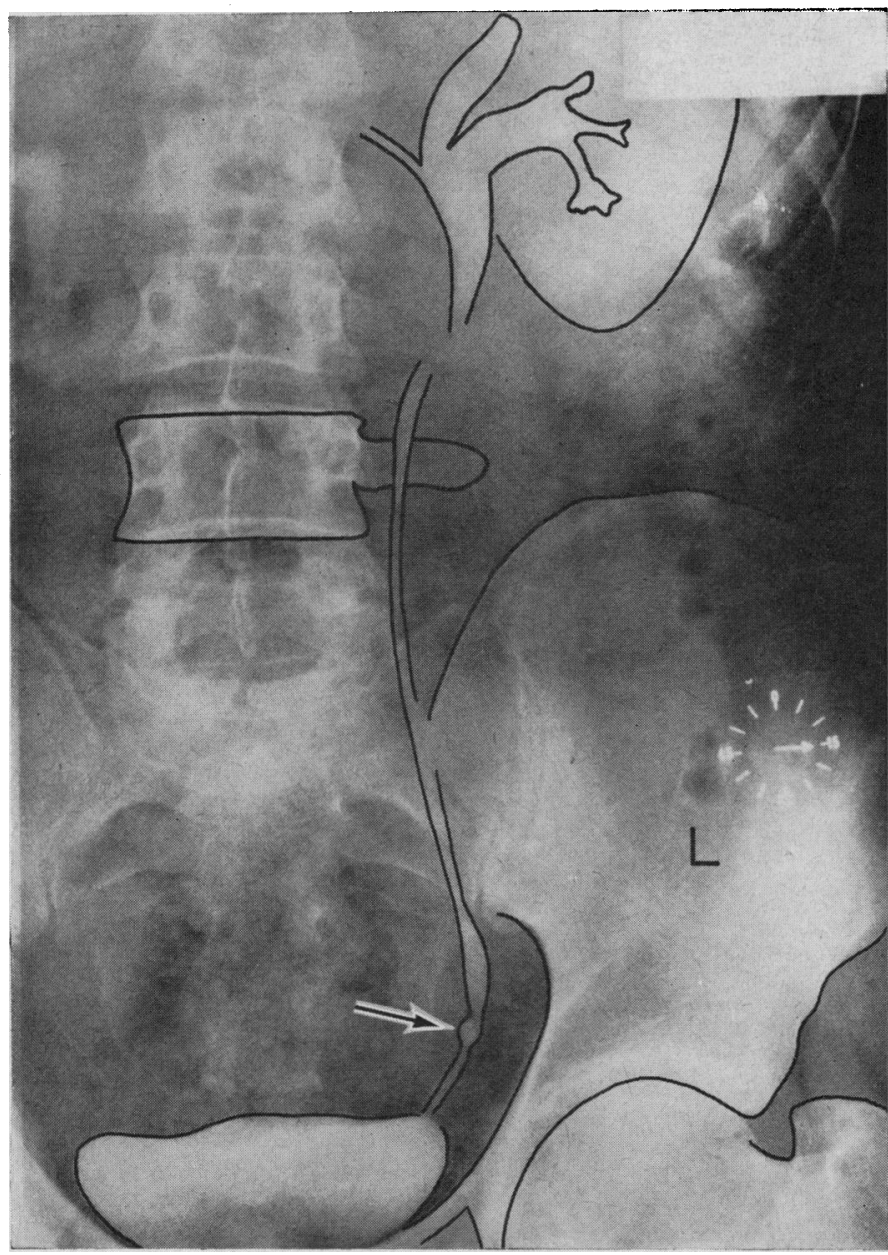

Fig. 1.-Acute urogram. Obstructed left kidney; ureter outlined; calculus in ureter.

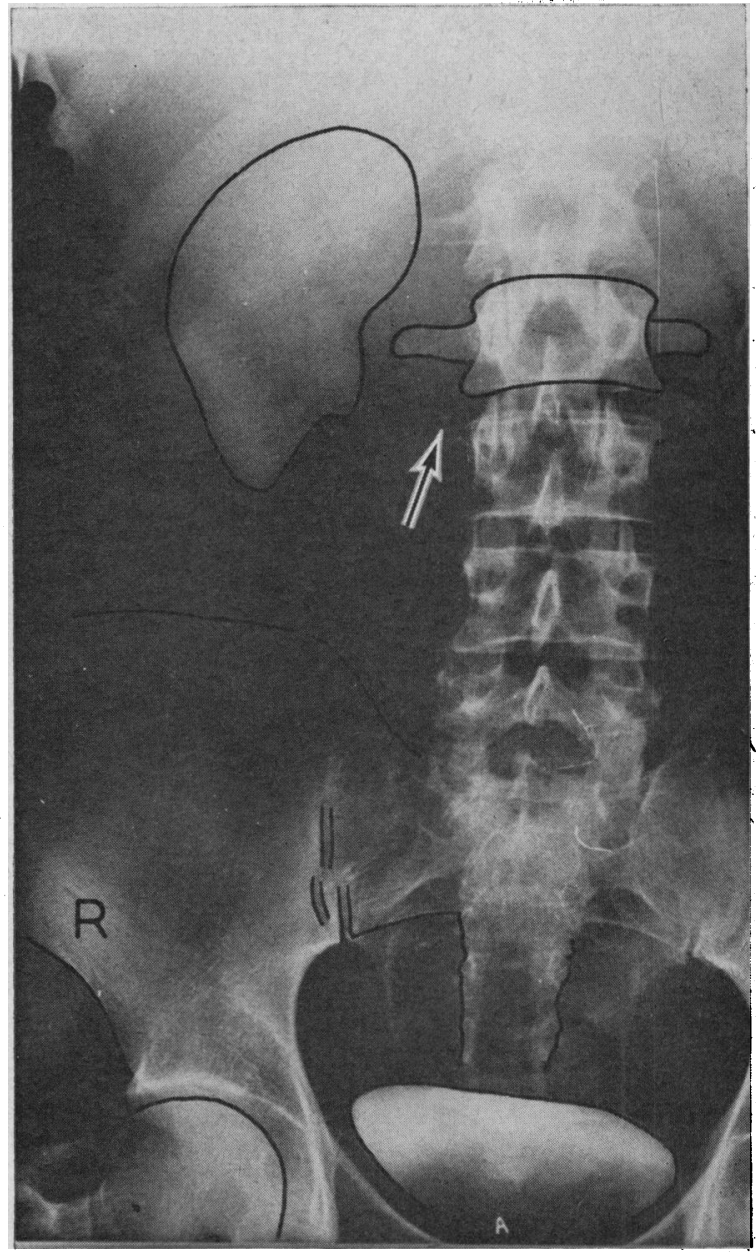

FIG. 2.-Acute urogram. Obstructed kidney (nephrogram); ureter not outlined; opacity in line of ureter.

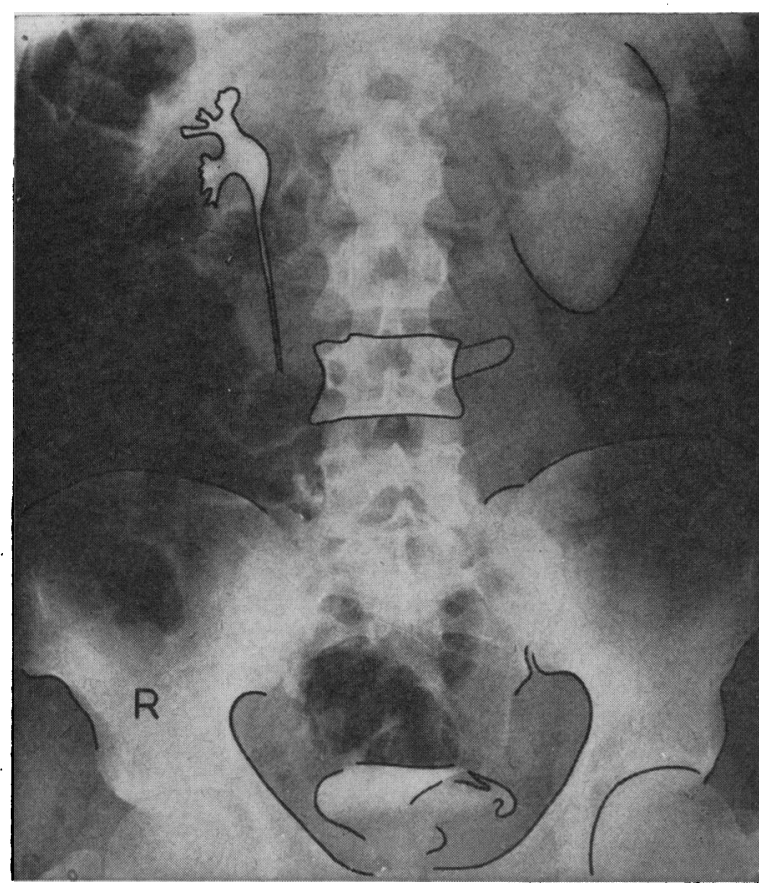

FIG. 3.-Acute urogram. Obstructed left kidney (nephrogram); ureter not outlined; no opaque shadow in line of ureter.

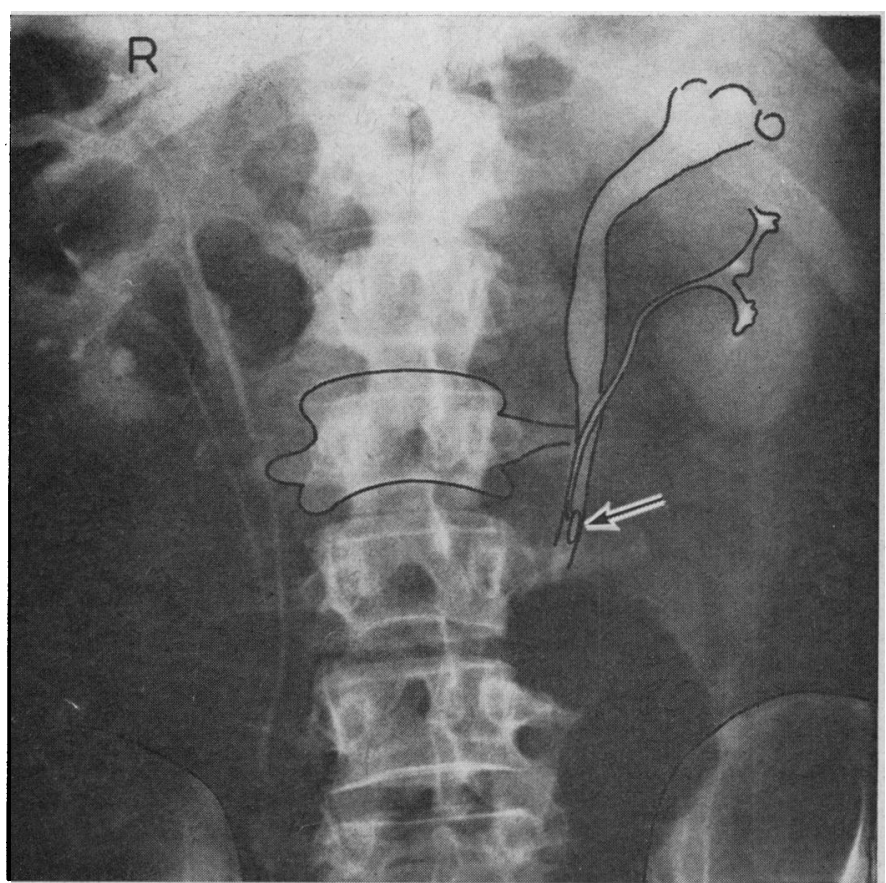
FIG. 4.-Acute urogram. Calculus producing obstruction in upper ureter
of duplex kidney (left). 


\section{R. D. W. MCLEAN ET AL.: ACUTE UROGRAPHY}

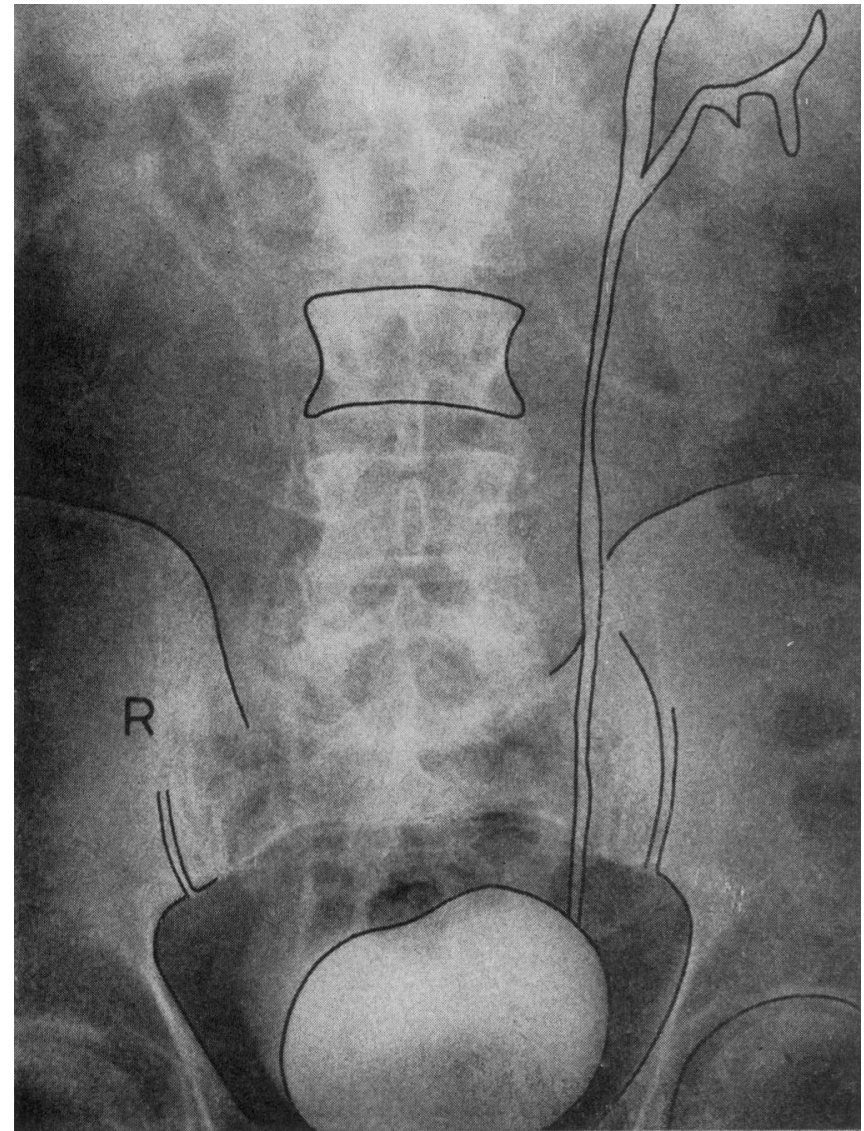

FIG. 5

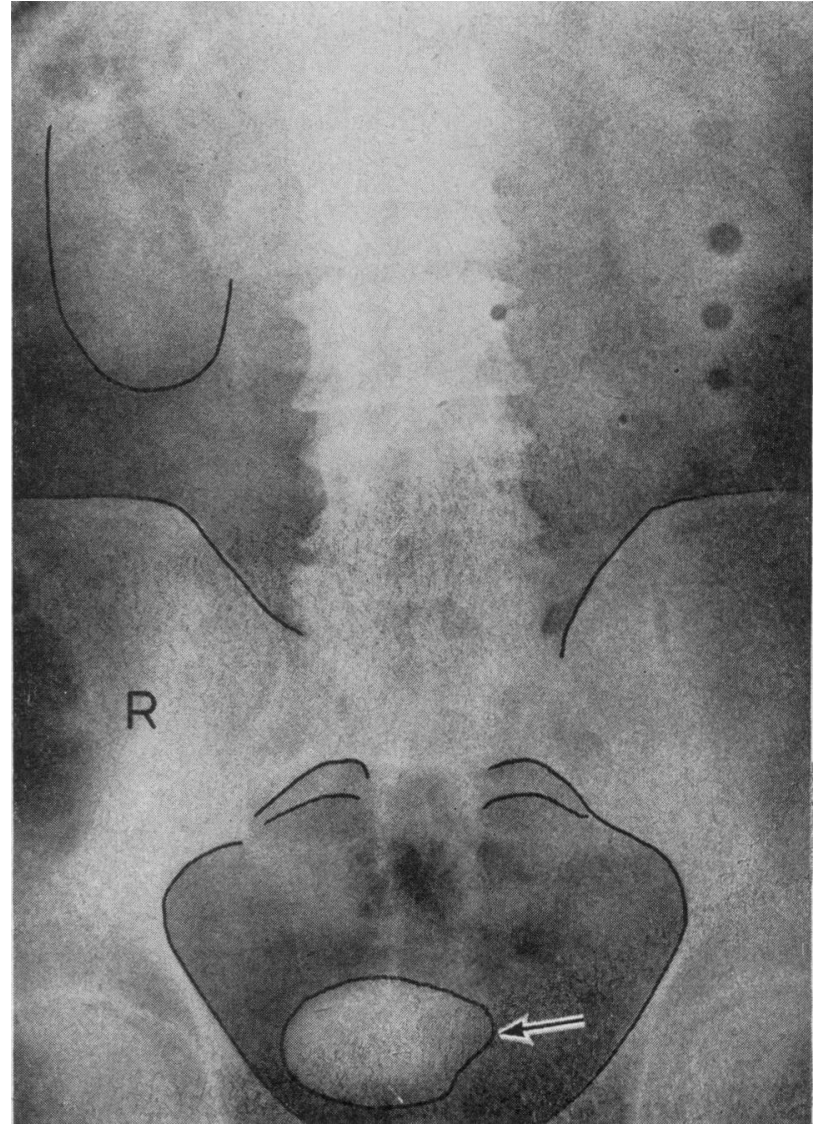

Fig. 6

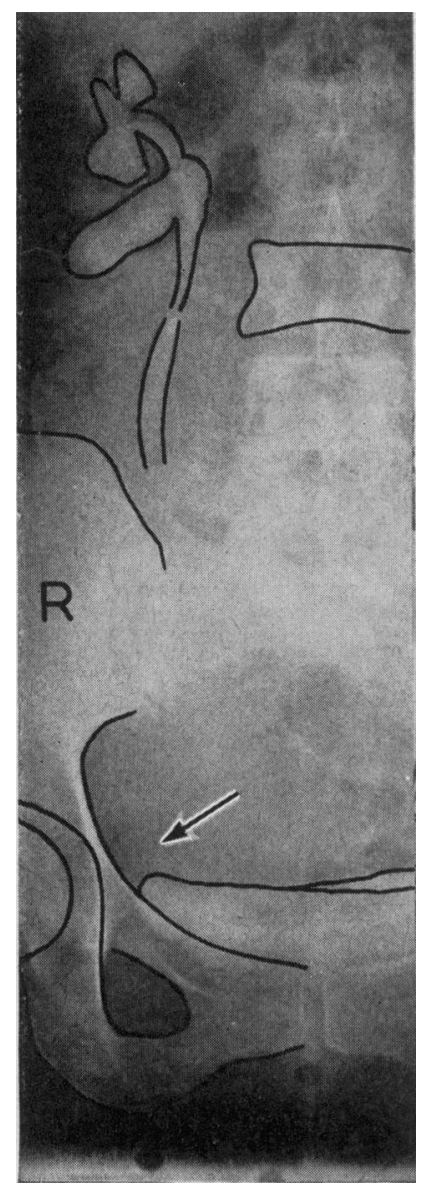

Fig. 7

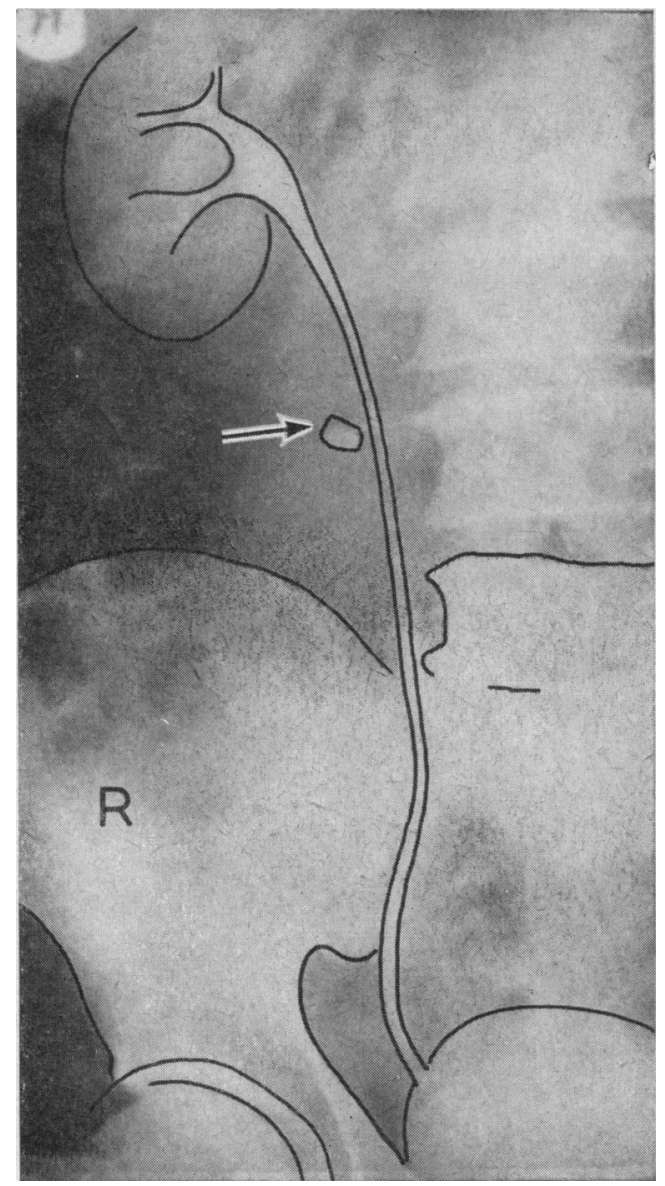

FIG. 8
FIG. 5.-Normal I.V.P., following uretero-lithotomy.

FIG. 6.-Acute urogram. Carcinoma of bladder; obstruction to left ureteric orifice presenting as renal colic.

FIG. 7.-Acute urogram. 20-week primigravida, showing dilatation of right ureter and pelvis, with calculus at lower end of ureter.

Fig. 8.-Acute urogram. Suspected calculus lies outside line of ureter. (Proved to be perforation of duodenal ulcer.) 


\section{R. D. W. MCLEAN ET AL.: ACUTE UROGRAPHY}

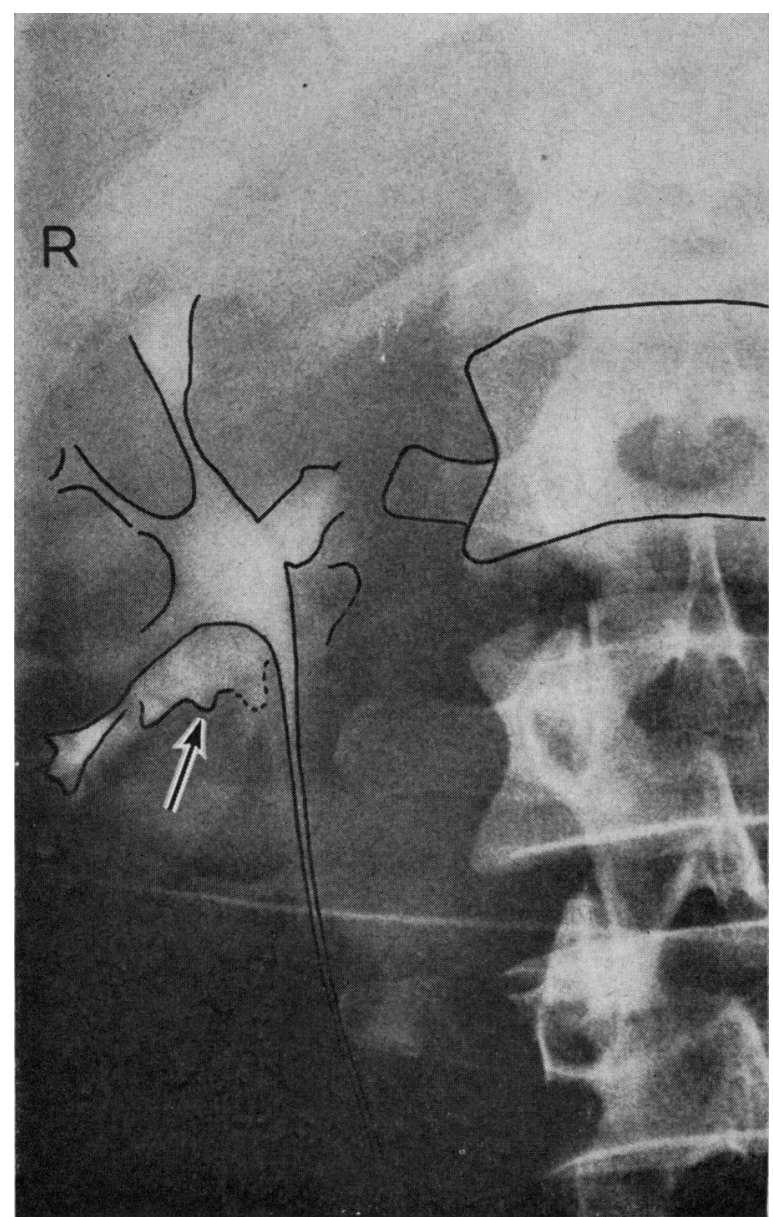

Fig. 9

FIG. 9.- Acute urogram. Renal trauma, leading to intrarenal extravasation of contrast (right).

FIG. 10.-14 days later-normal I.V.P.

FIG. 11.-Acute urogram. Bladder diverticulum presenting as haematuria.

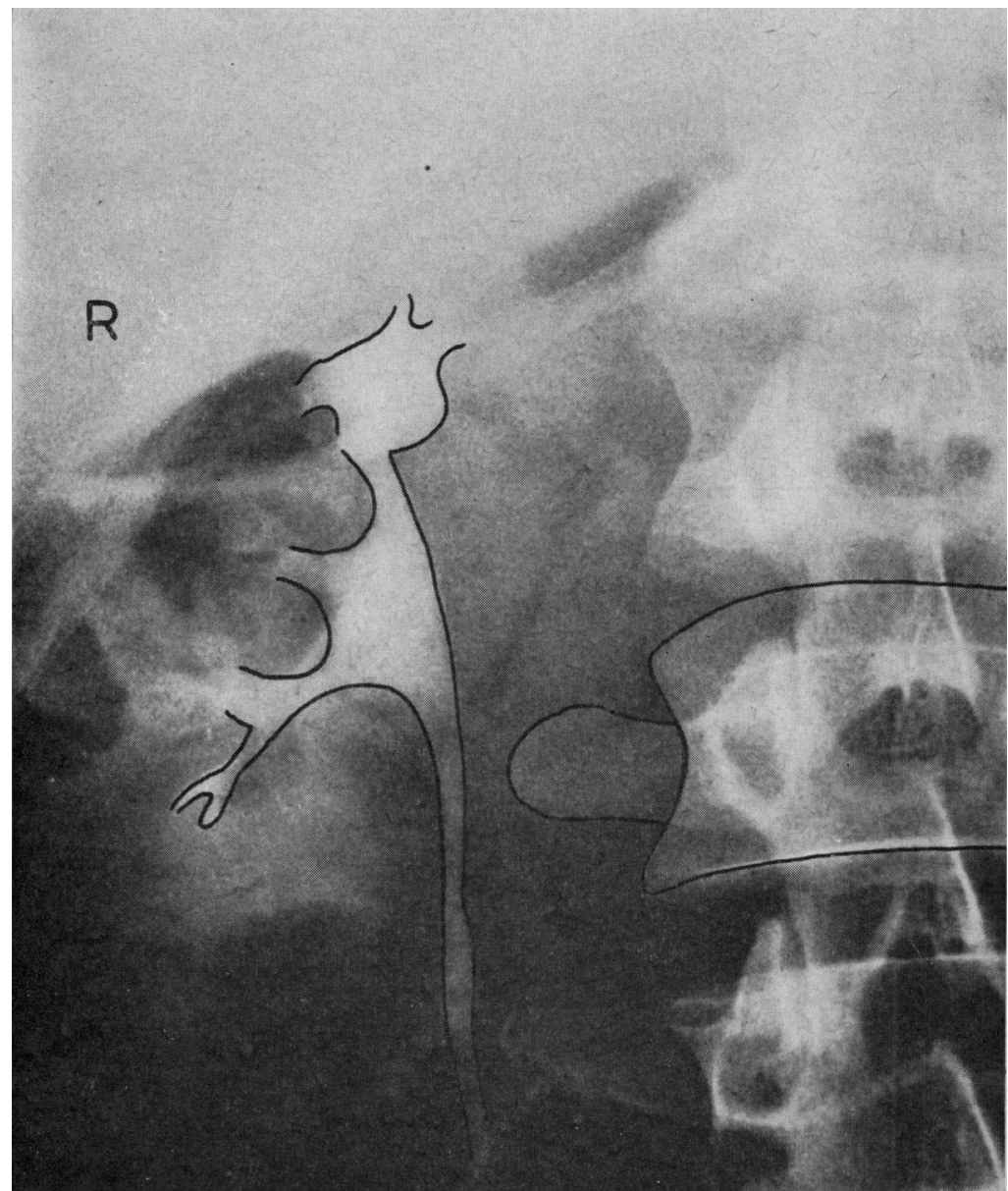

Fig. 10

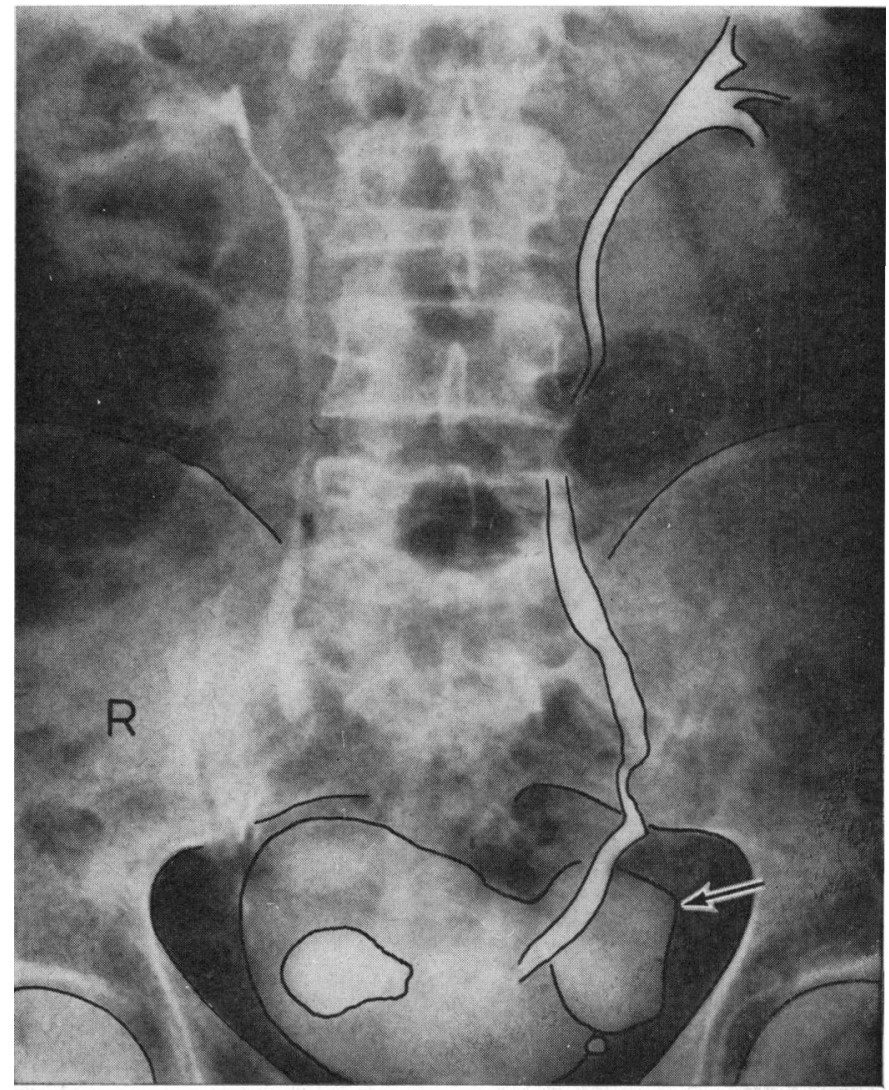

Fig. 11 
urography was delayed 24 hours $42 \%$ of cases had normal urograms. Smith (1966) found that a delay of one to seven days resulted in $62 \%$ of urograms being negative. This may lead to doubt about the original diagnosis, and subsequently to unnecessary investigations.

Illustrative Case.-A 28-year-old man presented with severe intermittent pain in the right iliac fossa for eight hours before admission. He had vomited twice, and had no other urinary symptoms. He had been admitted to hospital on four previous occasions with a similar history. On the first two occasions a diagnosis of renal colic was made, but a standard intravenous pyelogram on the day after admission showed no abnormality. On the third admission a normal appendix was removed. On the fourth, after a negative intravenous pyelogram, barium enema, and cystoscopy, the possibility of neurosis was considered. On this fifth admission an acute urogram showed an obstructed kidney and a small calculus at the lower end of the right ureter. This passed spontaneously.

Comment.-It seems probable that the previous symptoms were due to small renal calculi. Had acute urography been performed the diagnosis would have been made and a series of further investigations avoided.

Acute urography proves of value in cases where urgent diagnosis and treatment are necessary (Special Plate, Figs 4-6).

Illusiratre Cases.-A 53-year-old woman was admitted to hospital with severe pain in the right renal angle and a temperature of $101^{\circ} \mathrm{F}$. $\left(38.3^{\circ} \mathrm{C}\right.$.). Fifteen years previously she had had a left nephrectomy for pyelonephrosis. On examination she looked toxic ; the right kidney was enlarged and tender. An acute urogram showed marked back-pressure changes in the right kidney with the calculus lying at the lower end of the right ureter. This was removed by a Dormia extractor. The patient made an uneventful recovery.

A 50-year-old man presented with a two-year history of intermittent pain in the left renal angle. An I.V.P. was carried out on three occasions elsewhere, and on each occasion he was informed there was no lesion noted on the left side, but that he had a congenital abnormality on the right. On presenting at this hospital an acute urogram showed a calculus obstructing the ureter draining the upper half of a duplex kidney; the duplex anomaly was also present on the right (Fig. 4). The calculus was removed by uretero-lithotomy. A check radiograph one month later showed normal function on the left side (Fig. 5).

Comment. It is interesting that non-function of the upper half of this duplex kidney during standard pyelography led to a missed diagnosis; it became apparent during acute urography.

\section{Pregnancy}

The differential diagnosis of abdominal pain during pregnancy may be difficult, yet radiological investigation, because of the dangers of radiation, has to be kept to a minimum. This method of acute urography is well suited to pregnancy when a stone is suspected and failure of conservative management demands location of the calculus and removal. To minimize radiation we have modified the technique to taking one radiograph 12 minutes after injection of the medium. Three patients in the series developed renal colic during pregnancy which failed to respond to conservative management. Acute urography by this method satisfactorily demonstrated kidney obstruction and a ureteric calculus in all three. One required pyelolithotomy and the other two calculi were removed by a Dormia extractor.

Illustrative Case.-A primigravida aged 31, who was 20 weeks pregnant, developed severe right abdominal and right renal angle pain. She had passed a urinary calculus three years previously. Tenderness was present in both the right iliac fossa and the right kidney area. She was treated conservatively with analgesics and antispasmodics. Over the next $\mathbf{4 8}$ hours pain became more severe and unresponsive to analgesics. An acute urogram showed an obstructed kidney on the right side, and a calculus at the lower end of the right ureter. This was removed by Dormia extractor (Special Plate, Fig. 7). She had no further urinary symptoms and the pregnancy continued uneventfully until term.

\section{Value of Normal Urogram in " Renal Colic"}

Nine patients in this series suspected clinically of having renal colic were found to have normal urograms. This led to reconsideration of the diagnosis (see Table).

Illustrative Case.-A 44-year-old man was admitted to hospital with a history of having been awakened by severe pain in the right side of his abdomen eight hours previously. The pain had eased before admission but was present as a "dull ache." He had passed renal calculi in 1957 and 1959 . He had a history suggestive of duodenal ulceration for 10 years. On examination tenderness was present along the right side of the abdomen. A provisional diagnosis of renal colic was made on the strength of clinical findings and a history of previous calculi. This was supported by a plain $x$-ray examination of the abdomen arranged by his own doctor, which showed a small opacity near the tip of L 4 . An acute urogram, however, showed a normal kidney on the right side, the opacity lying outside the ureter. At laparotomy a perforated duodenal ulcer was found with leakage of fluid along the right paracolic gutter (Special Plate, Fig. 8).

\section{Renal Trauma}

In cases of haematuria following renal trauma the main value of acute urography lies in demonstrating the presence or absence of a functioning kidney on the contralateral side, should nephrectomy have to be considered. The urographic appearances also have prognostic value. Five patients had normal urograms, and in them haematuria gradually diminished over the next 48 hours. In the other two cases structural damage to the kidney was demonstrated, and haematuria persisted for over two weeks.

Illustrative Case.-A 48-year-old man presented with haematuria following a car accident. On examination a mass was palpable in the right kidney area. Acute urography demonstrated intrarenal extravasation of the medium on the right side, suggesting fracture of the renal substance (Special Plate, Fig. 9). On conservative management his general condition improved and haematuria ceased after two weeks. The abdominal mass had gradually subsided and a repeat urogram at this stage showed only minimal leak of contrast. A check intravenous pyelogram 14 days later showed normal appearances (Special Plate, Fig. 10).

Nunn (1962) has shown that in $50 \%$ of cases with intrarenal extravasation of the medium conservative management is successful, but that in all cases with perirenal extravasation nephrectomy was necessary.

A normal urogram in patients admitted with trauma may prove of value in distinguishing between a ruptured kidney and rupture of the spleen or liver.

Illustrative Case.-A 39-year-old man was admitted to hospital after a road traffic accident. His main injuries were to the left lower chest and left renal area, and he had profuse haematuria. On examination a slightly tender mass was palpable in the left hypochondrium. The tenderness was localized and there was no evidence of peritoneal irritation elsewhere in the abdomen. Over the next four hours the pulse rate rose from 80 on admission to 130, and he developed signs of shock. An acute urogram showed a normal renal tract on the left side, enabling a diagnosis of ruptured spleen to be made ; a transabdominal approach revealed a ruptured spleen with localization of blood by the omentum.

\section{Haematuria}

Cystoscopy performed at the time of haematuria is often useful in locating the bleeding to one or other of the kidneys. Occasionally, however, no haematuria is present at the time of cystoscopy. In these circumstances an acute urogram, performed before cystoscopy, may be useful in locating the bleeding to one or other kidney and so enable further investigation, such as retrograde pyelography, to be done at that time if thought necessary. Of seven cases in the series presenting in this way the diagnosis was made on acute urography in three. 
Illustrative Case.-A 70-year-old man presented with haematuria. Acute urography demonstrated a large diverticulum of the bladder (Special Plate, Fig. 11), but at cystoscopy the orifice of the diverticulum could not be visualized owing to continuing haematuria from the site.

We are indebted to the radiographers, Altnagelvin Hospital, for their co-operation in carrying out these investigations. We acknowledge the help given by Mr. P. Farrell, group medical photographer.
REFERENCES

Boeminghaus, H. (1932). Arch. klin. Chir., 171, 109.

Elkin, M. (1963). Radiology, 81, 484.

Goy, I. C. (1963). 7. Coll. Radiol. Aust., 7, 39.

Heilbrun, N., and Chittenden, G. (1946). Radiology, 47, 51.

Hellmer, H. (1935). Acta radiol. (Stockh.), 16, 51.

Helimer, H. (1962) Aust. N.Z. 7 Surg. 31, 263

Ross, J. A. McNair, T. J., Isaac, A., Thomson, W. N., and High, D. (1962). F. roy. Coll. Surg. Edinb., 8, 70.

Smith, I. (1966). Brit. F. Surg., 53, 93.

Wesson, M. B., and Fulmer, C. C. (1932). Amer. F. Roentgenol., 28, 27.

Woodruff, S. R., and Lewis, W. F. (1941). F. Urol., 45, 303.

Wulf, H. B. (1935). Acta radiol. (Stockh.), 16, 77.

\title{
Self-certification for Brief Spells of Sickness Absence
}

\author{
P. J. TAYLOR,* B.SC., M.D., M.R.C.P., D.I.H.
}

Brit. med. F., 1969, 1, 144-147

Summary : An arrangement is described for manual $\checkmark$ workers of an oil refinery to certify their own short spells of sickness absence instead of obtaining a medical certificate. No serious abuse has been found, and these spells of absence are more evenly spread throughout the week than those certified by a doctor. This system can help to reduce both the work-load of practitioners and the lost time in industry.

\section{Introduction}

The obligation to provide certificates of incapacity for work has long been a cause of complaint from the medical profession. The procedure for National Insurance certification was simplified in 1966, and an absence of up to seven days can now be covered by one form instead of two.

With the rise in national sickness absence rates (Office of Health Economics, 1968) the overall burden of certification is increasing, and, in addition, more firms are introducing sickpay schemes which generally require a medical certificate to authorize payment (Ministry of Labour, 1964). It is becoming the practice of such firms to accept "sight" of the National Insurance certificate instead of requiring a separate piece of paper, and this can reduce the extra load of the practitioner.

The demand for certificates to cover brief spells of absence has been the main cause of objection, since most of them can only be ipse dixit in nature, and the B.M.A. Annual Representative Meeting in 1964 resolved that "every effort should be made to abolish the demand for medical certificates of incapacity for illness of less than three days."

Uncertificated paid sick absence for periods of up to three days has been usual in industry for staff (white collar) grades, but is still rare for manual workers (labour). A few firms have followed the example of the Civil Service, which allows "Whitley Days," but this is unlikely to be widely extended.

This paper describes the effects of introducing a signed declaration for short spells of sick absence among manual workers who had previously been required to produce a medical certificate.

\section{Description of Certification Requirements}

The company has operated a relatively generous sick-pay scheme covering all employees since 1946. Payment is made without waiting days and consists of the full normal wage after allowing for the individual's entitlement to social security

* Seniar Medical Officer, Shell U.K. Limited, Shell Haven, Stanford-le-
Hope, Essex. Present address: Institute of Oocupational Health, Hope, Essex. Present address : Institute of Oocupational Heal
London School of Hygiene, 43 Russell Square, London W.C.1. benefit. While staff have always been allowed to take up to three working days on the strength of their own statement of incapacity, manual employees were required to provide a doctor's certificate.

Early in 1964, in order to obtain some form of diagnostic data for staff short-sickness absences, a " self-certificate" was introduced, which staff who had been absent were asked to complete. This arrangement was voluntary, but with explanation and encouragement a description of symptoms is now obtained for all such uncertified absences.

In June 1965 a similar scheme was introduced for the manual hourly-paid labour. Medical certificates are no longer required for sickness absences of up to three working days, but on

\section{CONFIDENTIAL}

To: 1. Time Office

2. Refinery Medical officer, Medical Centre.

Please fold to this Iine

\section{UNCERTIFIED SICKNESS REPORT}

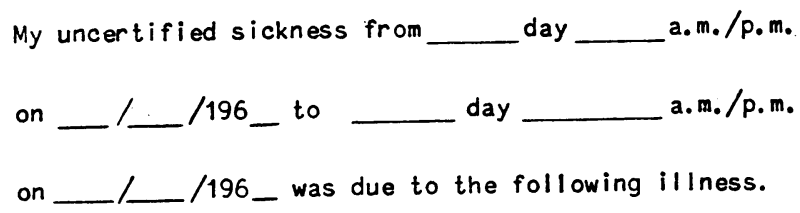

(Brief description of symptoms-e.g. cold, 'flu, diarrhoea, sore throat).

Date Signed

S.P. No._Department 